\title{
A three dimensional FEM-DEM technique for predicting the evolution of fracture in geomaterials and concrete
}

\author{
Francisco Zárate ${ }^{\mathrm{a}, \mathrm{b}, *}$, Alejandro Cornejo ${ }^{\mathrm{a}, \mathrm{b}}$, Eugenio Oñate ${ }^{\mathrm{a}, \mathrm{b}}$ \\ ${ }^{a}$ Centre Internacional de Mètodes Numèrics en Enginyeria (CIMNE) \\ ${ }^{b}$ Universitat Politècnica de Catalunya (UPC), \\ C. Gran Capitan s/n, Campus Nord, 08034 Barcelona, Spain.
}

\begin{abstract}
This paper extends to three dimensions (3D) the computational technique developed by the authors in 2D for predicting the onset and evolution of fracture in a finite element mesh in a simple manner based on combining the finite element method (FEM) and the discrete element method (DEM) approach [23]. Once a crack is detected at an element edge, discrete elements are generated at the adjacent element vertexes and a simple DEM mechanism is considered in order to follow the evolution of the crack. The combination of the DEM with simple 4noded linear tetrahedron elements correctly captures the onset of fracture and its evolution, as shown in several 3D examples of application.

Keywords: Discrete elements, finite elements, fracture mechanics, FEM-DEM technique
\end{abstract}

\section{Introduction}

Many authors have contributed different techniques to define the growth of a fracture in a brittle or ductile continuum $[8,11,20,21]$. One of the latest works defines the continuum by means of discrete elements [19]. However, the inherent difficulty for calibrating the material parameters in the DEM, as well as the need for a large number of discrete elements $[11,19]$ question it effectiveness,

*email: zarate@ cimne.upc.edu 
even though the qualitatively results of the DEM for predicting multiple fracture patterns are pretty good.

This paper extends to 3D analysis the basis of the simple FEM-DEM procedure proposed by the authors for predict the onset and propagation of fractures in solids [23]. The method extends a well-defined crack opening methodology termed Element Elimination Technique (EET) [10, 16, 22] that creates discrete elements at the crack lips. Some important aspects inherent to the formulation here presented guarantee the good results obtained, like a smoothed stress field, mass conservation and the use of a simple algorithm to ensure the post-fracture contact between the fractured edge. The FEM-DEM approach proposed is applied to a collection of benchmark problems in 3D which evidence the good performance of this numerical technique for nonlinear analysis of solids involving multi-fracture situations.

\section{3D Implementation}

Following the same FEM-DEM philosophy defined for the 2D case [23], the fundamental ideas have been exported to the 3D analysis. Basically the FEMDEM procedure can be grouped in five steps. Each of these will be discussed in more detail in the next sections.

- FEM discretization with 4-noded tetrahedra.

- Stress field definition.

- Elemental damage definition.

- DEM discretization.

- Substepping time integration of the dynamic equations for the DEM and FEM regions.

In short, the solution strategy starts by evaluating the transient mechanical behaviour of a finite element (FE) mesh of 4-noded tetrahedra that discretize a solid. The mesh connectivities emulates the discrete element (DE) links were damage is evaluated. The mechanism to transfer an element damage to the DE links is defined by the different cutting planes for each tetrahedron. In this way the element stiffness, affected by the damage, can be defined in a standard form and a new iteration can be made. 
When the elemental damage is greater than a certain value, it is considered that the element stiffness is so degraded that it is possible to eliminate the element following the Element Elimination Technique (EET) $[10,16,22]$. At this time, new discrete elements are created at the vertices of the removed tetrahedron. In this way a crack is formed in the continuum discretized by FE with the crack lips defined by the DE. It is interesting that as the crack grows and branches, some discrete elements can be detached from the FE mesh when the element that holds a DE is eliminated, creating a disgregation of the continuum.

\subsection{FEM discretization with 4-noded tetrahedral}

Much research has been spent in recent years in the development of the FEM for modelling the onset and propagation of cracks in frictional materials $[4-6,9$, 15]. However, most FEM procedures for crack prediction require sophisticated element formulations, as well as remeshing in the vicinity of the possible cracks paths $[9,15]$.

The approach followed in this paper uses the FEM to model a continuum whose fracture is described by means of DE, as cracks appear.

The simple 4-noded tetrahedra is chosen for the 3D FEM solution. The analysis domain is initialliy discretized with a tetrahedral mesh, just as in a standard 3D analysis with the FEM. A simple damage model is introduced assuming a progressive degradation of the element stiffness as described below.

\subsection{Stress field definition}

Having a proper stress field is key for define the right damage level and the crack path. In the $2 \mathrm{D}$ problem, the stress field is defined by smoothing the stress values at the elemental Gauss point in a new point located at the middle of the sides of each triangular element. This strategy follows the superconvergent patch recovery method proposed by Zienkiewicz, and Zhu [24] which overcomes the need to add stabilization terms to the stress field, as in alternative procedures [4-6].

Since the 3D stress field between elements is discontinuous, the same smoothing mechanism is used. That is, the stresses on each tetrahedral element edge are computed as the arithmetic mean of the stresses inside all elements that share that edge.

Like for the 2D case [23], the Rankine failure criteria has been used for predicting the onset of fracture in brittle materials [5,13]. During failure an exponential damage law is considered to progressively degrade the material stiffness [7]. The evolution of the damage parameter $d$ (varying from 0 to 1 ) is defined as 

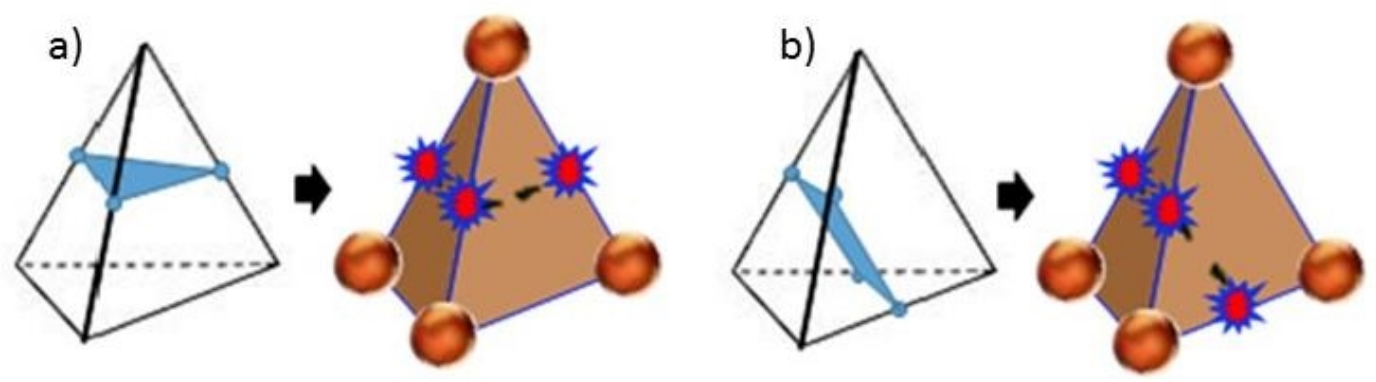

Figure 1: Cutting planes in a tetrahedron. a) One node disconnect b) Two nodes disconnected.

$$
d=1-\frac{\sigma_{f}}{\sigma_{I}} \exp \left[A\left(1-\frac{\sigma_{I}}{\sigma_{f}}\right)\right] \quad \text { with } \quad A=\left[\frac{G E_{o}}{l \sigma_{f}^{2}}-\frac{1}{2}\right]^{-1}
$$

where $\sigma_{I}$ is the major principal stress, $l$ is the edge lenght, $\sigma_{f}$ is the tensile strength, $G$ is the fracture energy and $E_{o}$ the undamaged Young modulus of the material.

\subsection{Elemental damage definition}

Once damage has been evaluated at each element edge, which corresponds to an virtual DE link, the elemental is calculated as the maximum damage created at any cut plane existing within the tetrahedron (Figure 1). On each tetrahedron there are four cut planes that disconnect one node from the other three nodes (Figure 1a) ) and three cut planes that disconnect two nodes from the other two nodes (Figure 1b) ).

The cutting plane damage is defined as the average damage value of each edge that the cutting plane intersects. If the elemental damage exceeds a certain threshold, then the tetrahedron is completely removed $[10,16,22]$ and new discrete elements are created at the associated nodes maintaining the mass of the element removed. In many cases is usual that the elemental damage threshold is reached at the same time steep for all the elements that shares a side. In this case the crack grows along the removed finite elements and the crack lips are defined by discrete elements.

\subsection{DEM discretization}

When a tetrahedron FE is fully removed (i.e. the element stiffness is neglected) four discrete elements (or particles) are created at the element node. In our work 
we have used spheres for representing the discrete elements. The mass of each new discrete element corresponds to the nodal mass and its radius is the maximum one that guarantees the contact between the adjacent discrete elements without creating any overlapping between them. Other algorithms can be used for generating DE [11,12], but good results have been achieved with the proposed one.

Once a DE is created, the forces at the contact interfaces are used to define the interaction of the element with the adjacent ones. These forces are due only to the contact interaction in the normal and tangential directions. At the contact point, the minimum radius of the particles in contact are used to evaluate the contact forces [19].

For 3D problems the normal contact force $F_{n}$ at a contact point between two spheres is given by:

$$
F_{n}^{i j}=\frac{A^{i j}}{d_{i j}} E_{o} u_{n} \quad \text { with } \quad A^{i j}=\pi r_{c}^{2}
$$

where $r_{c}$ is the radius of the smaller of the two particles interacting at the interface $i j$. $\quad u_{n}$ is the normal overlap between the two discrete elements and $d_{i j}$ is the distance between the element centres.

The tangential force $F_{s}$ is decomposed in two orthogonal directions $s_{1}$ and $s_{2}$ contained at the plane normal to the direction $d_{i j}$. For each direction the normal force is given by:

$$
F_{s_{i}}=\min \left\{\begin{array}{l}
u_{s_{i}} \frac{E_{o}}{2(1+v)} \\
\mu F_{n}\left(\frac{u_{s_{i}}}{u_{s}}\right)
\end{array}\right.
$$

were $\mu$ is the friction coefficient, $v$ is the Poisson's ratio, $u_{s_{i}}$ is the relative tangential displacement between two discrete elements and $u_{s}$ is the module of the $\boldsymbol{u}_{s}$ vector. More details of Eqs. (2) and (3) can be found in [19].

As we pointed out in [23], the total number of DE generated in an analysis is only a small fraction of the number of nodes of the mesh. Therefore, the searching algorithm for evaluating the contact interactions between discrete elements does not consume much computational resources.

\subsection{Substepping time integration of the dynamic equations for the DEM and FEM regions}

One of the main problems in 3D FEM analysis is the large number of equations that arise from discretization. Adding to these the difficulties to consider 


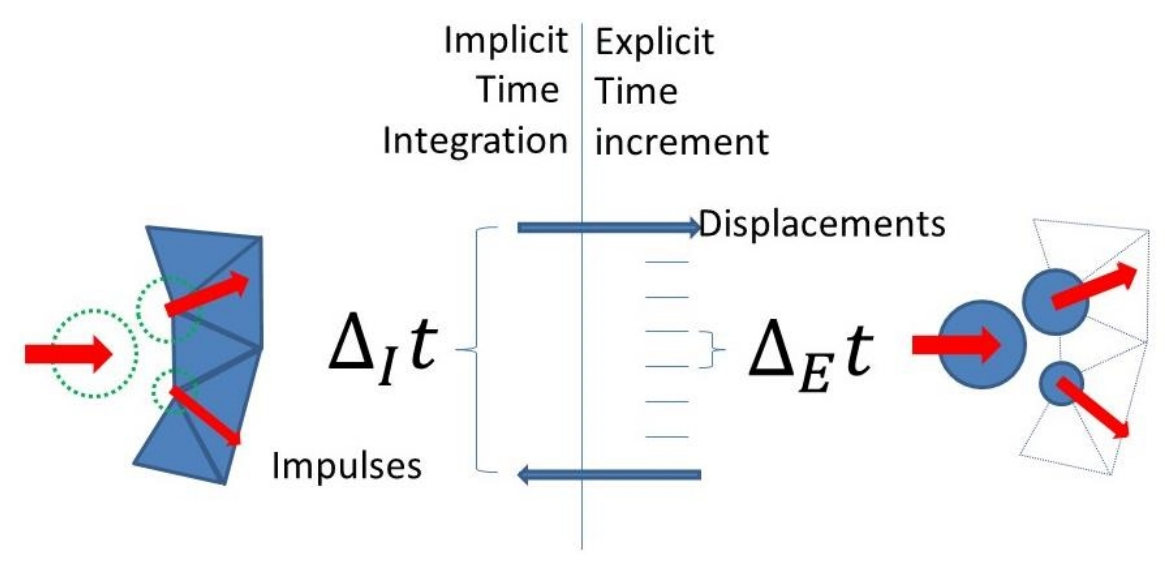

Figure 2: Substepping time scheme used for FEM and DEM time integration.

a large time increment, an implicit time integration is the best solution strategy. However, this type of integrations is not typically applied when discrete elements are used because it is very difficult to correctly identify and quantify the contacts and contact forces between these elements.

The implementation followed in this work corresponds to a time stepping scheme in which the FEM solution is calculated using an implicit Newmark scheme, while for the discrete elements computation we use an explicit centred differences scheme. Usually the implicit time increment is 100 times greater than the explicit one.

The advantage of this strategy is that the number of discrete elements is much lower than that of finite elements, so the explicit integration performed in an implicit time step is quite fast. Before beginning a new time step $\Delta_{I} t$ in the implicit scheme, the same time period is analysed with an explicit scheme over the discrete elements using a time increment of $\Delta_{E} t$.

The contact between discrete elements is quantified by the sum of the impulses that occur along the analysis during $\Delta_{I} t$. This effect is expressed as a force applied on the element at time $t+1$ for computing the implicit solution, as shown in Figure 2. In this way the FEM-DEM solution advances in time.

It has been observed that it is not advisable for the time steps $\Delta_{E} t$ and $\Delta_{I} t$ to have a ratio greater than 1:500 since there may be some discrepancy between the explicit and implicit solutions. Having this in mind, the strategy solution implemented yields excellent results, as shown in the examples presented in next section. 


\section{EXAMPLES}

In this section several examples are shown in order to show the good behavior of the 3D FEM-DEM strategy described. The first example presented is the 3D study of a normalized sample for a tensile test. The second example corresponds to the indirect tensile test also known as Brazilian test widely used in in concrete and rock mechanics. The third example consists of a concrete shear test proposed in [14]. Finally, we present the seismic analysis of the central nave of the church located at the Poblet monastery. In this example a $6 M_{w}$ earthquake is applied to highlight the effect of the opening and closing of the cracks generated.

\section{1. $3 D$ normalized tensile test}

The first example corresponds to the fracture analysis of a flat concrete specimen under tensile stress. The geometry is defined according to the norm D638 of the American Section of the International Association for Testing Materials (ASTM) [1] as shown in Figure 3 where the three meshes of 4-noded tetrahedra used and the boundary conditions can be seen. A constant horizontal velocity is imposed to the two ends of the specimen.

The study has been performed using the 3D FEM-DEM technique previously described. In order to localize the fracture, only one band of elements is allowed to break at the failure stress level corresponding to the tested material, using the linear damage model previously described. The results obtained are analysed by plotting the displacements of points PA and PB shown in Figure 3.

The Young modulus, the Poisson's ratio and the density are respectively $E_{o}=$ $30 \times 10^{9} \mathrm{~Pa}, v=0.2, \gamma=1.0 \times 10^{3} \mathrm{~N} / \mathrm{m}^{3}$, the tensile strength $\sigma_{f}=10 \times 10^{3} \mathrm{~Pa}$ and the fracture energy $G_{2}=7.5 \times 10^{-3} \mathrm{~J} / \mathrm{m}^{2}$.

The specimen deforms by applying a constant velocity of $0.5 \times 10^{-} 7 \mathrm{~m} / \mathrm{s}$. at both tips of the specimen. Figure 4 shows the damaged geometry. Note that where fracture appears, discrete elements are created at the crack lips as explained in the previous sections. Due to the DE sizing algorithm which guarantees that the DE creation do not generate spurious contact forces, the element radius depends on the ordering in which the DE are generation order.

The displacement of points $\mathrm{PA}$ and $\mathrm{PB}$ are at the right and at the centre of the specimen, respectively (Figure 3) are tracked in order to evaluate the crack opening. Figure 5 shows the load-displacement relationship at these points. For

the three meshes considered the displacement evolution is very similar and in agreement with the expected results [23]. 


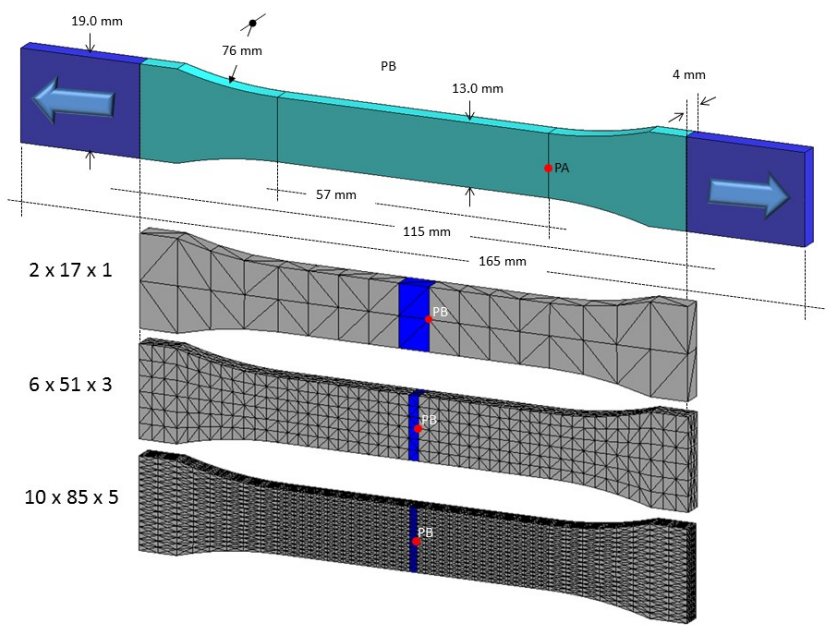

Figure 3: Normalized tensile test of a 3D concrete specimen. Finite element mesh and dimentions acoording to ASTM D638 norm.

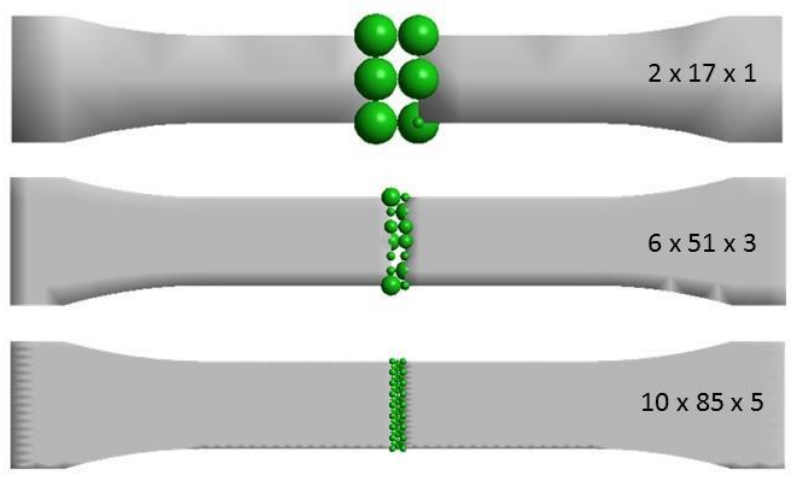

Figure 4: Normalized tensile test of a concrete specimen. Cracked zone with the discrete particles generated for the three finite element meshes considered. 


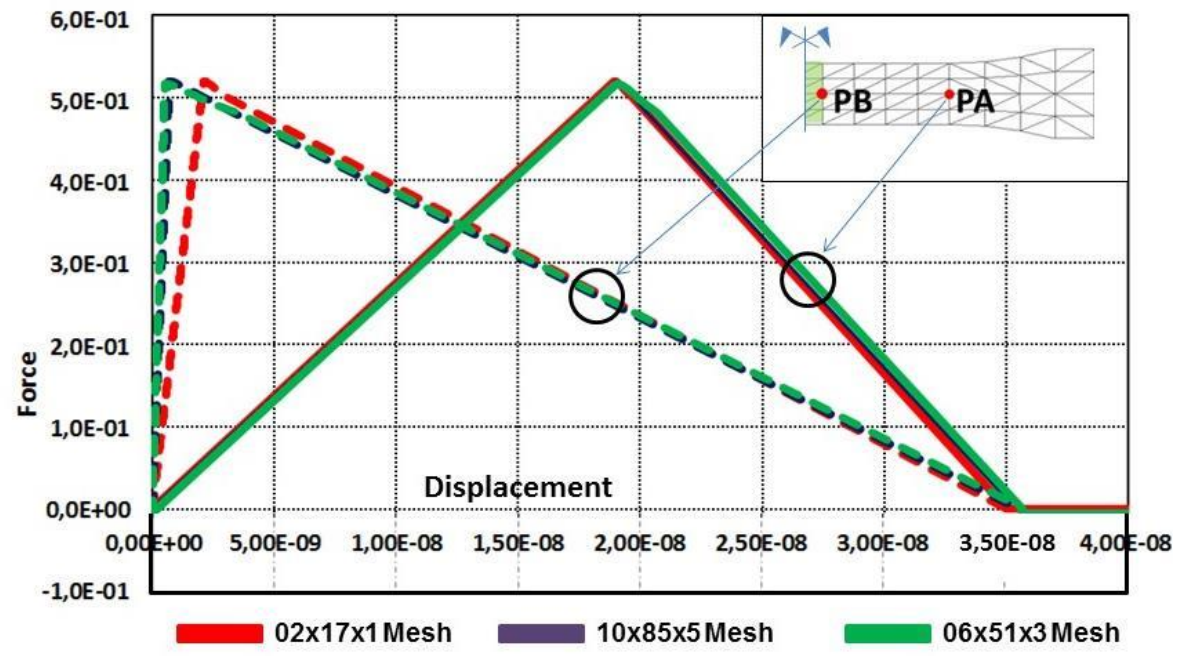

Figure 5: Normalized tensile test of a concrete specimen. Load-displacement at points PA and PB of the specimen using $G_{2}=7.5 \times 10^{-3} \mathrm{~J} / \mathrm{m}^{2}$

Since the fractured elements have a different size for each mesh, the displacement of point $\mathrm{PB}$ in the elastic region becomes smaller as the element size is reduced. Beacause the crack opening is a fast phenomenon, the use of an implicit integration scheme does not allow to capture properly the movement of the analysed points. However, it follows the theoretical results.

We point at the insensitivity of the force-displacement curves to the quality of the mesh. This is a distinct feature of the FEM-DEM procedure [17, 18,23]

\subsection{Indirect tensile test}

The Brazilian tensile strength (BTS) test is a very practical and simple way to evaluate the tensile strength of concrete and rock materials. The concrete sample analysed is a cylinder of $0.2 \mathrm{~m}$ diameter $(D)$ and $0.1 \mathrm{~m}$ thickness $(t)$, which is a diametrically loaded by a press. The tensile strength value is computed by the following relationship [3]:

$$
\sigma_{\max }=\frac{2 P}{\pi t D}
$$

were $P$ is the applied load.

The material properties are $E_{o}=21 \times 10^{9} \mathrm{~Pa}, v=0.2, \gamma=7.8 \times 10^{3} \mathrm{~N} / \mathrm{m}^{3}$, $\sigma_{f}=10 \mathrm{KPa}$ and $G=1 \times 10^{-3} \mathrm{~J} / \mathrm{m}^{2}$. This corresponds to a maximum failure load of $P=314.16 N$. 


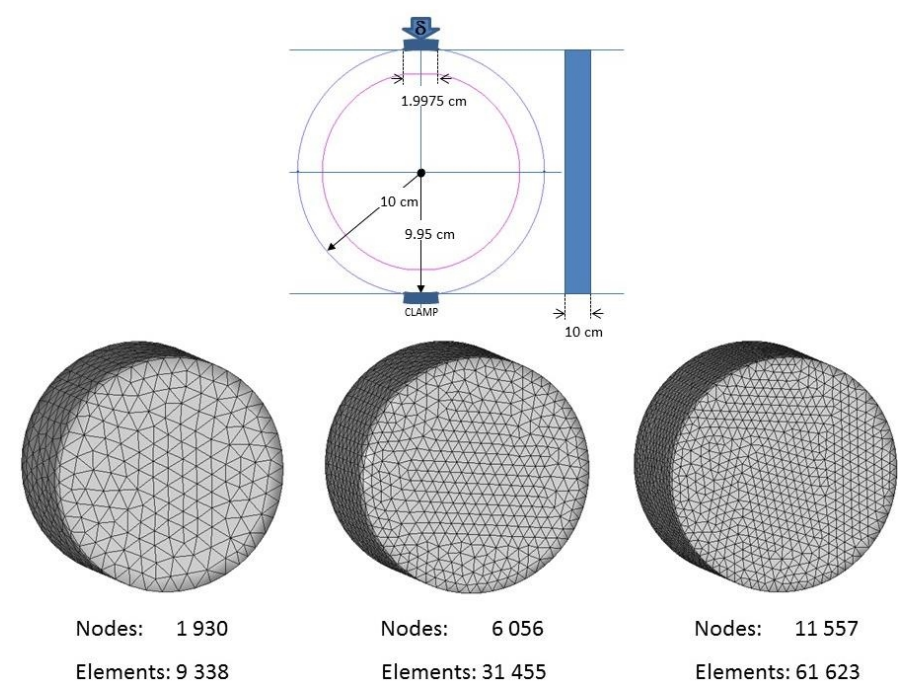

Figure 6: BTS test on a concrete specimen. Dimensions, boundary conditions and finite element meshes of three noded triangles used.

Three meshes of 9338, 31455 and 61623 4-noded linear tetrahedra elements each are used for the analysis, as shown in Figure 6. The sample is deformed by imposing a constant vertical velocity at the top of the sample.

Figure 7 depicts the crack and the discrete elements generated. It can be seen that the cracking pattern is similar for the three meshes and in agreement with the expected result. The numerical results for the load-displacement curve are presented in Figure 8. The values obtained for the maximum tensile strength are of (coarse to fine) $10693 \mathrm{~Pa}, 10351 \mathrm{~Pa}$ and $10235 \mathrm{~Pa}$ which yields in the range of $6 \%$ to $2 \%$ error versus the expected value of $\sigma_{f}=10 \mathrm{KPa}$.

Again we note the insensitivity of the load-displacement curves to the mesh size.

\subsection{Shear test}

A shear test is designed to apply stress to a test sample so that it experiences a sliding failure along a plane that is parallel to the forces applied. Generally, shear forces cause one surface of a material to move in one direction and the other surface to move in the opposite direction so that the material is stressed in a sliding motion.

The procedure proposed to determine the shear resistance has been based on the shear test proposed by Luong [14]. This test consists in a circular tube of axis 

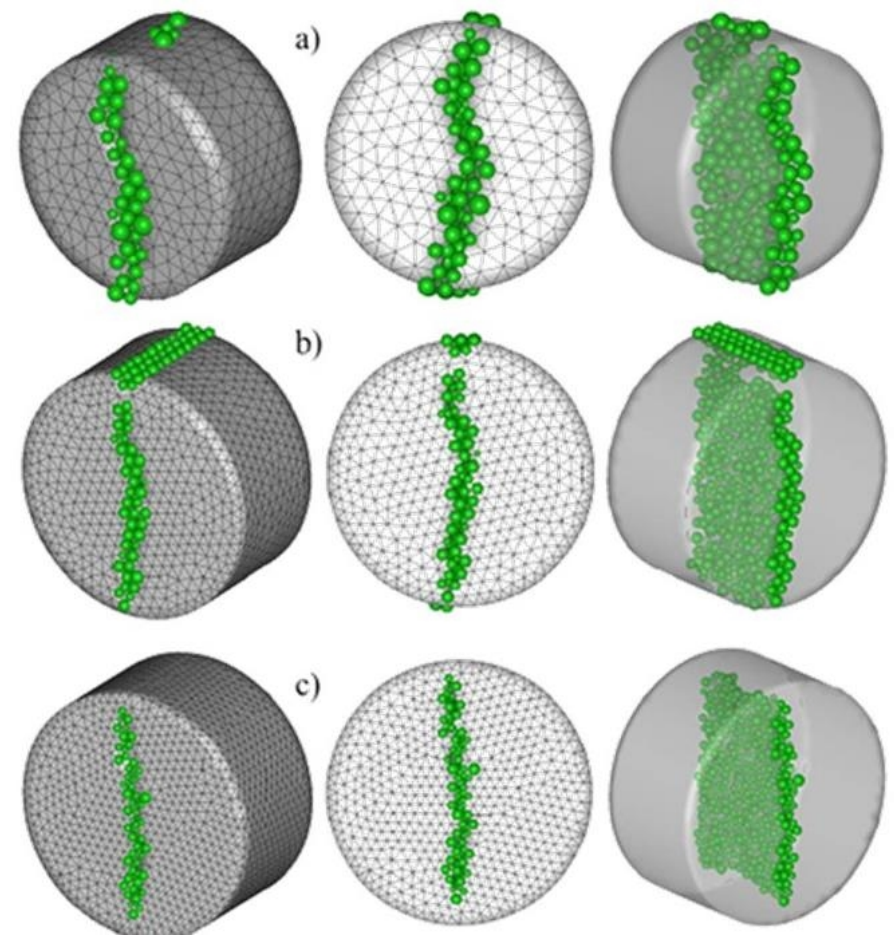

Figure 7: BTS 3D test on a concrete specimen. Damage zone and discrete elements generated. a) Coarse mesh, b) intermediate mesh, c) Fine mesh.

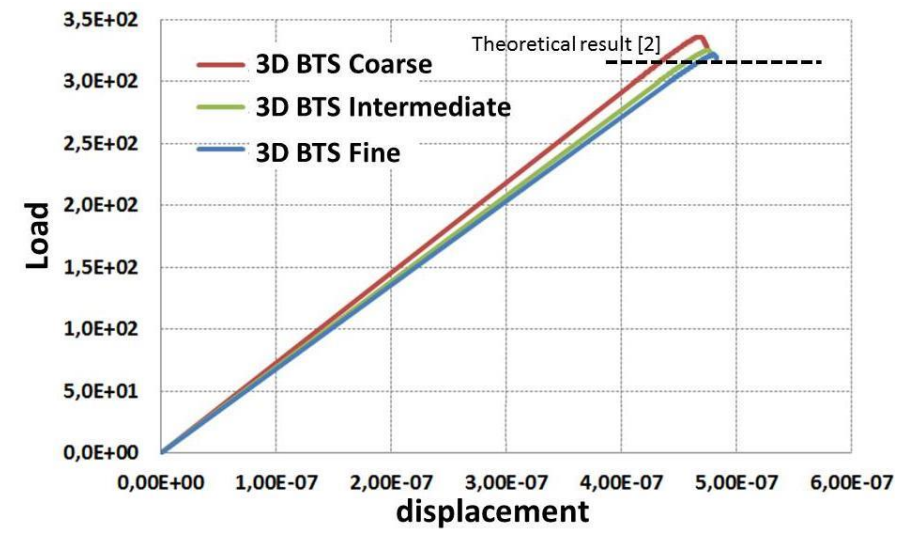

Figure 8: BTS 3D test on a concrete specimen. Force-displacement relationship for the three meshes used. 


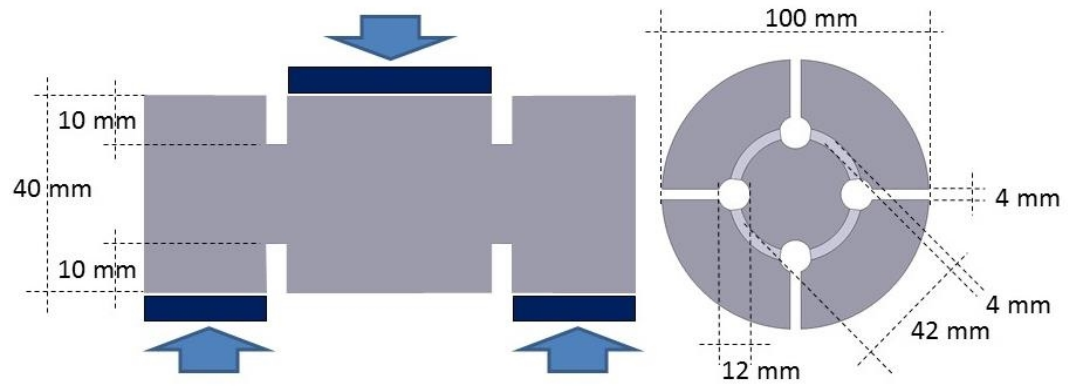

Figure 9: Shear test on a concrete specimen. Geometry and boundary conditions.
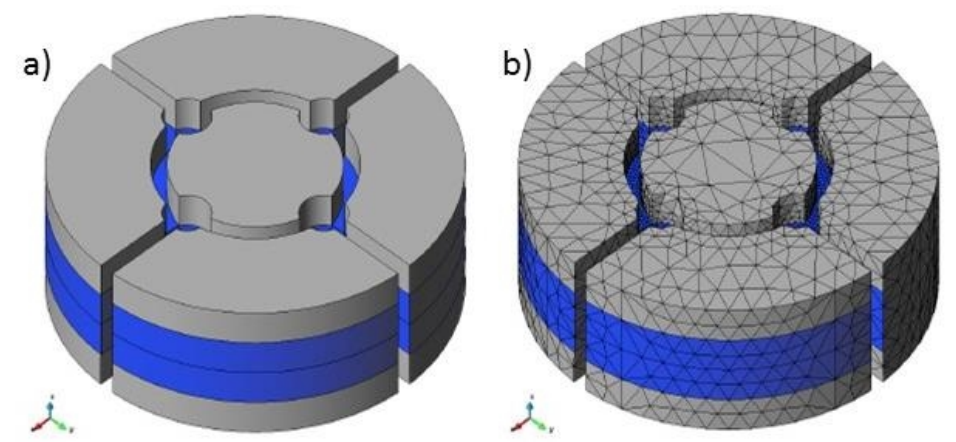

Figure 10: Shear test on a concrete specimen. a) CAD gemetry and b) finite element discrtetization.

$z=0$ subjected, on its internal and external faces, to a shear stress parallel to $z=0$ as it is described in Figure 9 were the boundary conditions can be deduced. The height of the notch is $10 \mathrm{~mm}$ whereas the width is $4 \mathrm{~mm}$. On the internal tube a constant vertical velocity of $1 \mathrm{~mm} / \mathrm{s}$ has been applied until failure.

The CAD geometry and the finite element mesh can be seen in Figure 10. The shear plane has been discretized with 87176 4-noded tetrahedral elements in order to capture properly the stress gradient at this zone.

The material properties are $E_{o}=35 \times 10^{9} \mathrm{~Pa}, v=0.22, \gamma=7.8 \times 10^{3} \mathrm{~N} / \mathrm{m}^{3}$, $\sigma_{f}=30 \mathrm{MPa}, \sigma_{t}=3 \mathrm{MPa}$ and $G=75 \times 10^{-3} \mathrm{~J} / \mathrm{m}^{2}$. In general, the shear strength of concrete is approximately $1 / 5$ or $1 / 6$ of the compressive stress [14].

Figure 11 shows the crack obtained by the numeric experiment and compared with the restored laboratory samples [14]. Figure 12 shows the force-displacement graph of the numerical test in which the initial elastic branch is well defined until the elements starts damaging and the stiffness decreases. From this Figure and 

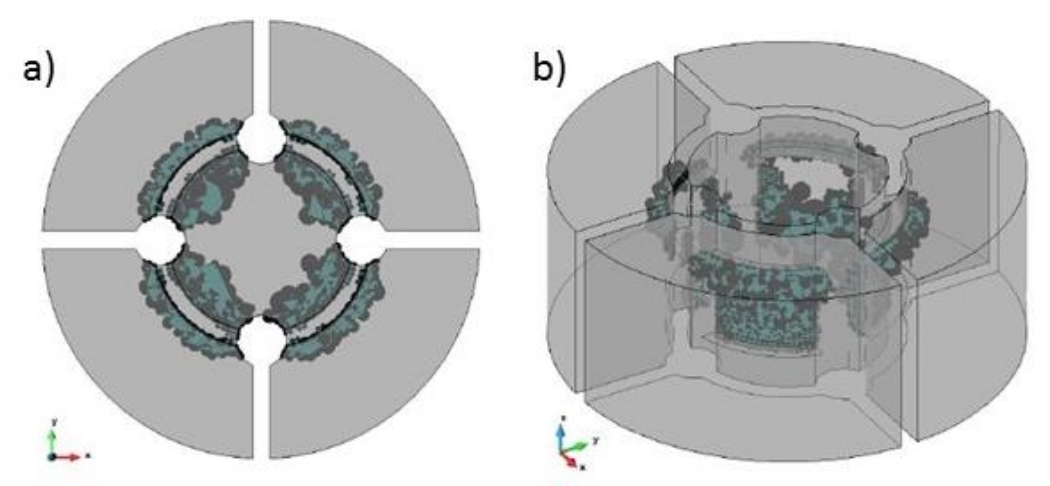

c)

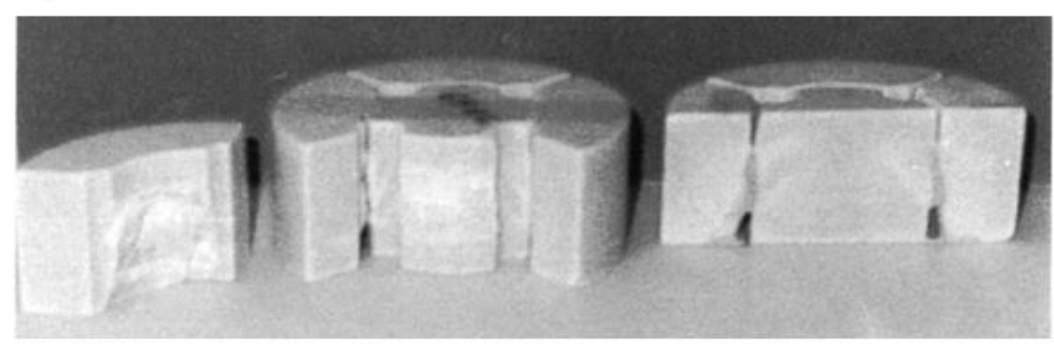

Figure 11: Shear test on a concrete specimen. a) Top view and b) perspective of the crack obtained at the numeric experiment. c) Restored laboratory samples [14].

according to the sample dimensions, the computed stress level reaches a value of 5.10MPa. The obtained result agrees in comparison with the expected value [14]. Figure 12 shows several laboratory samples, where the crack path obtained is similar to the one found using the FEM-DEM technique.

\subsection{Earthquake analysis of cistercian church}

The church belonging to the Royal Monastery of Santa Maria de Poblet, located in Vimbodí (Tarragona) is an historical artistic monument declared by UNESCO as a world heritage site. Built from the second half of the twelfth century, the temple adopts a basilical plant with its apse orientated to the East.

The Church is built on one nave plant and two aisles, with seven sections each. Including cruiser, central apse, abbatial chapels and ambulatory. The central nave has dimensions of $85 \mathrm{~m}$ long for $21 \mathrm{~m}$ wide and $28 \mathrm{~m}$ high, while the sides reach $18 m$ high. The height difference respect to the central nave is not solved by flying buttress of the gothic style, but with buttresses. The fully Romanesque central nave is covered with a ribbed pointed barrel vault, in each section as shown in 


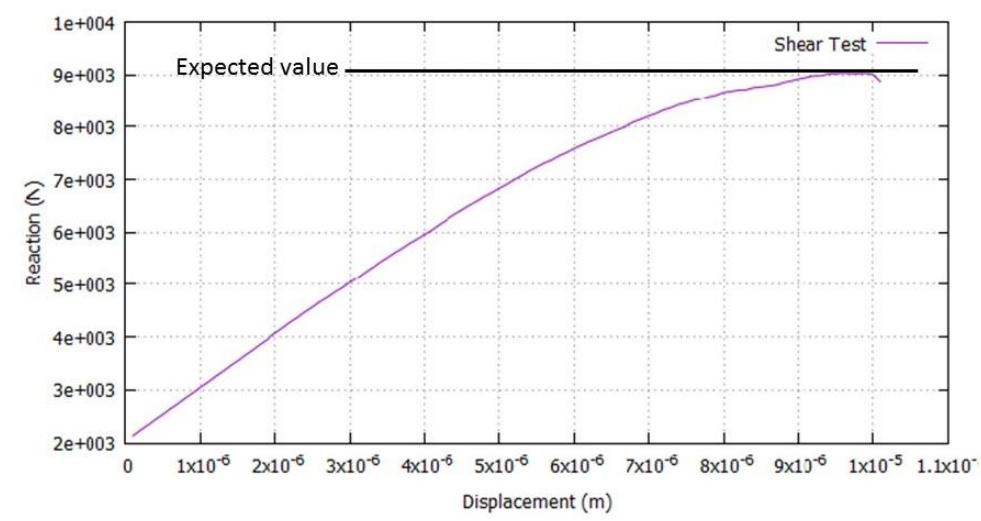

Figure 12: Shear test on a concrete specimen. Force-displacement relationship obtained with the FEM-DEM technique.

Figure 13.

In this case the solid domain is enveloped with invisible DE in order to solve the FE-DE contact. For simplifying reasons, only a section of the central nave is considered. This section is symmetrical respect the normal to the central nave direction. The FEM mesh has 9294 nodes and 32487 4-noded tetrahedra as shown in Figure $14 \mathrm{a}$ ). The material properties correspond to a limestone of the region, with $E_{o}=35 \times 10^{9} \mathrm{~Pa}, v=0.22, \gamma=25 \times 10^{3} \mathrm{~N} / \mathrm{m}^{3}, \sigma_{f}=780 \mathrm{MPa}, \sigma_{t}=5 \mathrm{MPa}$ y $G=100 \times 10^{-3} \mathrm{~J} / \mathrm{m}^{2}$. We consider the church to be at its clamped foundations.

The applied loads correspond to an imposed oscillatory movement, in the normal direction to the central nave axis as shown in Figure $14 \mathrm{~b}$ ). The values shown correspond to the oscillatory movement in the direction $\boldsymbol{S} 00 \boldsymbol{E}$ of the records in displacements obtained from the accelerogram of the earthquake "El Centro" occurred on May 18, 1940 [2]. These values are applied with a 50\% reduction to the model, so that it simulates a $6.0 M_{W}$ earthquake.

Although the record of movements lasts more than 50 seconds, the structure is only capable of standing on foot for 4 seconds, beyond this time the church fully collapses.

Figure 15 shows the cracks formed in the church at 1 second intervals. Initially the shear of the columns and the more rigid wall occurs, later, the arches bases are cutt off. From the second 5 it is observed how the structure fully collapses.

It is remarkable how the columns and arches of the aisles are held in place after they have been cut. This is due to the closure of the formed cracks. The FEM-DEM technique captures properly the contact between the different broken 


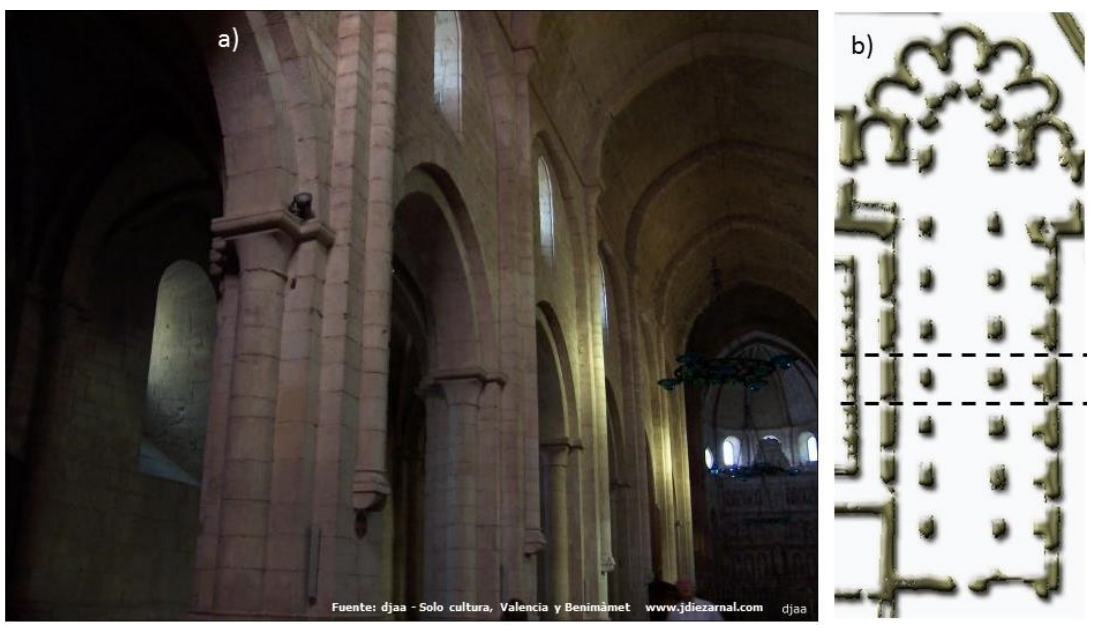

Figure 13: Cistercian church at the monastery of Poblet. a) Inner view of the central nave. b) Church plant and analized section.
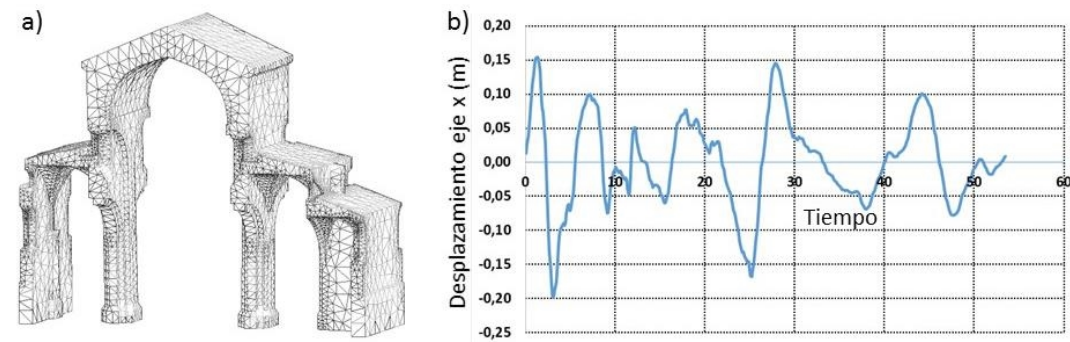

Figure 14: Cistercian church at the monastery of Poblet. a) FE Mesh of the analysed section. b) Dynamic exitation expresed in displacements. 


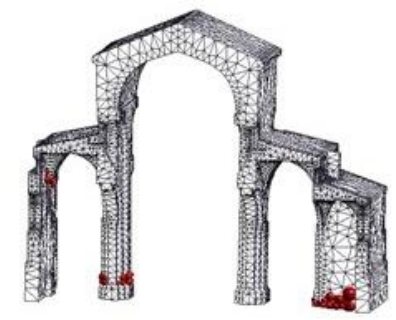

$$
\mathrm{t}=1.0 \mathrm{seg} \text {. }
$$

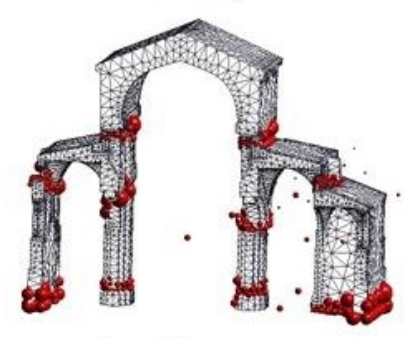

$t=4.0 \mathrm{seg}$.

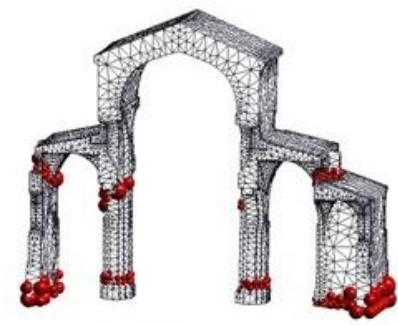

$t=2.0 \mathrm{seg}$.

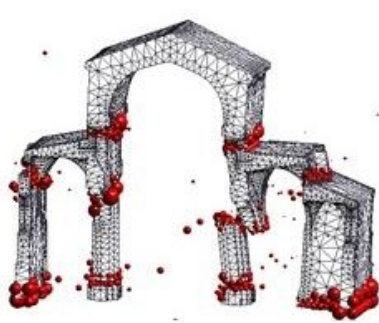

$\mathrm{t}=5.0 \mathrm{seg}$.

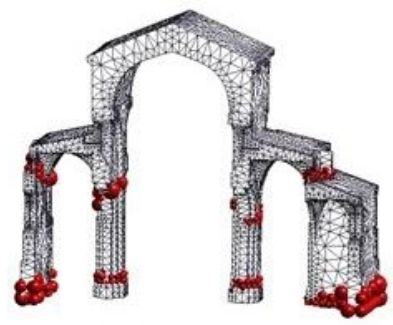

$\mathrm{t}=3.0 \mathrm{seg}$.

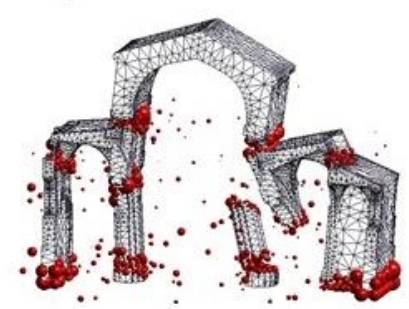

$t=6.0 \mathrm{seg}$.

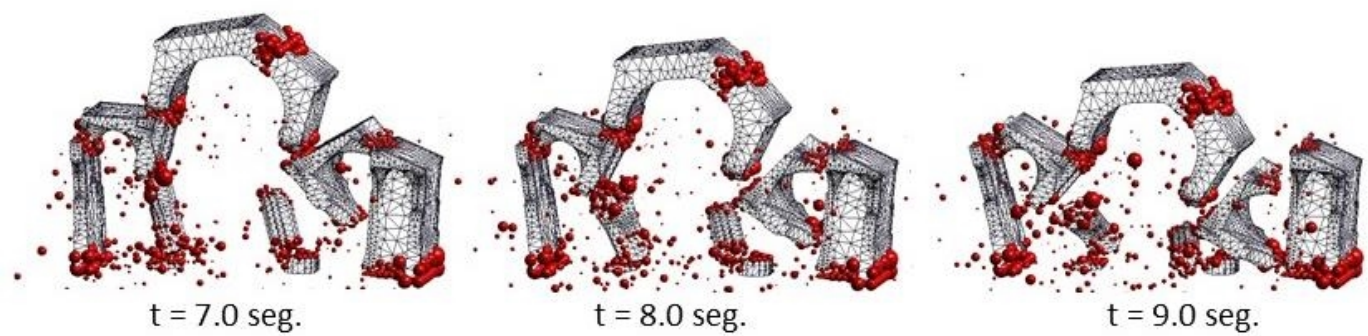

Figure 15: Cistercian church at the monastery of Poblet. Evolution of fractures at the central section of the nave for different times. 
elements of the structure. The fall of these elements occurs with a rotation as can be seen along seconds 5 to 9 in Figure 15.

\section{CONCLUSIONS}

A simple 3D FEM-DEM methodology has been proposed for analisys of the onset and evolution of the crack path in materials and structures. Excellent re-

sults have been found for the examples presented which confirms the predictive capability of the formulation.

\section{ACKNOWLEDGEMENTS}

This work was partially supported by the ICEBREAKER Proof of concept project of the European Research Council.

Results presented in this work have been obtained using the FEM2DEM and DEMPACK codes of CIMNE (http://www.cimne.com/dempack) were the DEMFEM methodology described has been implemented.

\section{References}

[1] Astm standard d638 - 10, 2003, "standard test method for tensile properties of plastics," astm international, west conshohocken, pa, 2003, doi: 10.1520/d0638-10, www.astm.org.

[2] Ground accelerogram from el-centro, imperial valley irrigation district (comp s00e). http://www.eng.ucy.ac.cy/petros/earthquakes/eq1.txt.

[3] F. L. L. B. Carneiro, A new method to determine the tensile strength of concrete, Proceedings of the 5 th meeting of the brazilian association for technical rules., 1943, pp. $126-129$. (In portuguese).

[4] M. Cervera, M. Chiumenti, and C. Agelet de Saracibar, Shear band localization via local j2 continuum damage mechanics, Comput. Methods Appl. Mech. Engrg. 193 (2004), 849 -880.

[5] M. Cervera, M. Chiumenti, and R. Codina, Mixed stabilized finite element methods in nonlinear solid mechanics part $i$ : Formulation., Computer Methods in Applied Mechanics and Engineering 199 (2010), $2559-2570$.

[6] __ Mixed stabilized finite element methods in nonlinear solid mechanics part ii: Strain localization., Computer Methods in Applied Mechanics and Engineering 199 (2010), 2571 -2589 .

[7] _ Mesh objective modelling of cracks using continuous linear strain and displacements interpolations, Int. J. Numer. Meth. Engng. 87 (2011), 962 -987.

[8] P. A. Cundall and O. D. L. Strack, A discrete numerical model for granular assemblies, Geotechnique 29 (1979), no. 1, $47-65$. 
[9] P.R. Johnson, N. Petrinic, and E. Sli, Element-splitting for simulation of fracture in $3 d$ solid continua, VIII International Conference on Computational Plasticity, Barcelona. (2005).

[10] S. Katagiri and S. Takada, Development of fem-dem combined method for fracture analysis of a continuos media, Memoirs of the Graduate School of Science and Technology, Kobe University Japan. 20A (2002 -03), $65-79$.

[11] C. Labra, Advances in the development of the discrete element method for excavation processes, Ph.D. Thesis, Barcelona, 2012.

[12] C. Labra and E. Oñate, High-density sphere packing for discrete element method simulations, Commun. Numer. Meth. Engng. 25 (2009), no. 7, 837 -849.

[13] J. Lopez, S. Oller, E. Oñate, and J. Lubliner, A homogeneous constitutive model for masonry, Int. J. Numer. Meth. Engng. 46 (1999), 16511671.

[14] M. Luong, Tensile and shear strengths of concrete and rock, Engineering Fracture Mechanics 1-3 (1990), no. 35, 127-135.

[15] L. Mishnaevsky Jr, N. Lippmann, and S.; Schmauder, Computational modelling of crack propagation in real microstructures of steels and virtual testing of artificially designed materials, International Journal of Fracture 120 (2003), 581 -600.

[16] A. Munjiza, The combined finite-discrete element method, isbn 0-470-84199-0, Wiley, 2004.

[17] E. Oñate, C. Labra, Zárate F., J. Rojek, and Miquel J., Avances en el desarrollo de los mtodos de elementos discretos y de elementos finitos para el anlisis de problemas de fractura, Anales de Mecnica de la Fractura 22 (2005), 27-34.

[18] E. Oñate, F. Zárate, M.A. Celigueta, J.M. González, J. Miquel, J.M. Carbonell, F. Arrufat, S. Latorre, and M. Santasusana, Advances in the dem and coupled dem and fem techniques in non linear solid mechanics, Advances in computational plasticity. oñate et al. (eds), springer, 2017.

[19] E. Oñate, F. Zárate, J. Miquel, M. Santasusana, M.A. Celigueta, F. Arrufat, R. Gandijota, K. Valiullin, and L. Ring, A local constitutive model for the discrete element method. application to geomaterials and concrete, Computational Particle Mechanics 2 (2015), 139-160.

[20] J. Rojek, E.. Oñate, F. Zarate, and J. Miquel, Modelling of rock, soil and granular materials using spherical elements, 2nd European Conference on Computational Mechanics ECCM2001, Cracow (2629 June 2001).

[21] J. Williams and R. O'Connor, Discrete element simulation and contact problem, Archives of Computer Methods in Engineering 6 (1999), no. 4, 279 -304.

[22] Shmauder S. Wulf J. and Fischmeister H.F., Finite element modelling of crack propagation in ductile fracture, Computation Materials Science 1 (1993), 297 -301.

[23] F. Zárate and E. Oñate, A simple femdem technique for fracture prediction in materials and structures, Computational particle mechanics 2 (2015), no. 3, 301-314.

[24] O.C Zienkiewicz and J.Z. Zhu, The superconvergent patch recovery (spr) and adaptive finite element refinement., Comput. Methods App. Mech. Engng. 101 (1992), 207 -224. 